Bull. Korean Math. Soc. 48 (2011), No. 6, pp. 1137-1146

http://dx.doi.org/10.4134/BKMS.2011.48.6.1137

\title{
ON THE RATES OF THE ALMOST SURE CONVERGENCE FOR SELF-NORMALIZED LAW OF THE ITERATED LOGARITHM
}

\author{
TIAN-XiaO PANG
}

\begin{abstract}
Let $\left\{X_{i}, i \geq 1\right\}$ be a sequence of i.i.d. nondegenerate random variables which is in the domain of attraction of the normal law with mean zero and possibly infinite variance. Denote $S_{n}=\sum_{i=1}^{n} X_{i}, M_{n}=$ $\max _{1 \leq i \leq n}\left|S_{i}\right|$ and $V_{n}^{2}=\sum_{i=1}^{n} X_{i}^{2}$. Then for $d>-1$, we showed that under some regularity conditions,

$$
\lim _{\varepsilon \searrow 0} \varepsilon^{2(d+1)} \sum_{n=1}^{\infty} \frac{(\log \log n)^{d}}{n \log n} I\left\{M_{n} / V_{n} \geq \sqrt{2 \log \log n}\left(\varepsilon+\alpha_{n}\right)\right\}
$$$$
=\frac{2}{\sqrt{\pi}(1+d)} \Gamma(d+3 / 2) \sum_{k=0}^{\infty} \frac{(-1)^{k}}{(2 k+1)^{2 d+2}} \text { a.s. }
$$

holds in this paper, where $I\{\cdot\}$ denotes the indicator function.

\section{Introduction and main results}

Let $\left\{X_{i}, i \geq 1\right\}$ be a sequence of i.i.d. random variables with common distribution function, and set $S_{n}=\sum_{i=1}^{n} X_{i}$ and $V_{n}^{2}=\sum_{i=1}^{n} X_{i}^{2}$ for $n \geq 1$, $\log x=\ln (x \vee e)$ and $\log \log x=\log (\log x)$. Hsu and Robbins [9] and Erdös [6] established the well-known complete convergence,

$$
\sum_{n=1}^{\infty} \mathrm{P}\left(\left|S_{n}\right| \geq \varepsilon n\right)<\infty, \quad \varepsilon>0
$$

if and only if $\mathrm{E} X=0$ and $\mathrm{E} X^{2}<\infty$. Baum and Katz [1] extended this result and proved that for $1 \leq p<2$ and $r \geq p$,

$$
\sum_{n=1}^{\infty} n^{r-2} \mathrm{P}\left(\left|S_{n}\right| \geq \varepsilon n^{1 / p}\right)<\infty, \quad \varepsilon>0
$$

Received March 22, 2010.

2010 Mathematics Subject Classification. 60F15.

Key words and phrases. almost sure convergence, self-normalized, domain of attraction of the normal law, law of the iterated logarithm.

Project supported by the National Natural Science Foundation of China (No. 11001236) and the Zhejiang Provincial Natural Science Foundation of China (No. Y6100091). 
holds if and only if $\mathrm{E} X=0$ and $\mathrm{E}|X|^{r p}<\infty$.

Many authors considered various extensions of the results of Hsu-RobbinsErdös and Baum-Katz. Some of them studied the precise convergence rates of the infinite sums as $\varepsilon \rightarrow 0$ (c.f. Heyde [8], Chen [2] and Spătaru [13]). But, this kind of results do not hold for $p=2$. However, by replacing $n^{1 / p}$ by $\sqrt{n \log \log n}$, Gut and Spătaru [7] established the following results called the precise asymptotics of the law of the iterated logarithm.

Theorem A. Suppose that $E X=0, E X^{2}=\sigma^{2}$ and $E X^{2}(\log \log |X|)^{1+\delta}<\infty$ for some $\delta>0$, and let $a_{n}=O\left(\sqrt{n} /(\log \log n)^{\gamma}\right)$ for some $\gamma>1 / 2$. Then

$$
\lim _{\varepsilon \searrow 1} \sqrt{\varepsilon^{2}-1} \sum_{n=1}^{\infty} \frac{1}{n} P\left(\left|S_{n}\right| \geq \varepsilon \sigma \sqrt{2 n \log \log n}+a_{n}\right)=1 .
$$

In the paper of Gut and Spătaru [7], the proof of Theorem A relies heavily on the Berry-Esseen inequality. Of late, by using a different method, that is, the strong approximation method (cf. Csörgö and Révész [3]), Zhang [14] provided the sufficient and necessary conditions of precise asymptotics of the law of the iterated logarithm not only for $S_{n}$, but also for $M_{n}$, here and in what follows, we denote $M_{n}=\max _{1 \leq i \leq n}\left|S_{i}\right|$.

On the other hand, it is well-known that the so-called self-normalized limit theorems put a totally new countenance upon classical limit theorems. The main advantage of self-normalization is that the limit theorems in probability and statistics, compared with the classical versions, may still hold under less or even without any moment condition (cf. Shao [12]) if the normalizing constants in the classical versions are replaced by an appropriate sequence of random variables. For a survey on recent developments in this area, the reader is referred to Lai and Shao [10] or de la Peña, Lai and Shao [5] for details.

Naturally, Pang, Zhang and Wang [11] studied the precise asymptotics in the self-normalized law of the iterated logarithm and below are their main results.

Theorem B. Let $\left\{X_{i}, i \geq 1\right\}$ be a sequence of i.i.d. nondegenerate random variables which is in the domain of attraction of the normal law with mean zero. Suppose $l(x):=E X_{1}^{2} I\left\{\left|X_{1}\right| \leq x\right\} \leq c_{1} \exp \left(c_{2}(\log x)^{\beta}\right)$ for some $c_{1}>0$, $c_{2}>0$ and $0 \leq \beta<1, \alpha_{n}=O(1 / \log \log n)$. Then, for $d>-1$, we have

$$
\begin{aligned}
& \lim _{\varepsilon \searrow 0} \varepsilon^{2(d+1)} \sum_{n=1}^{\infty} \frac{(\log \log n)^{d}}{n \log n} P\left(M_{n} \geq \sqrt{2 V_{n}^{2} \log \log n}\left(\varepsilon+\alpha_{n}\right)\right) \\
= & \frac{2}{\sqrt{\pi}(1+d)} \Gamma(d+3 / 2) \sum_{k=0}^{\infty} \frac{(-1)^{k}}{(2 k+1)^{2 d+2}}
\end{aligned}
$$

and

$$
\lim _{\varepsilon \searrow 0} \varepsilon^{2(d+1)} \sum_{n=1}^{\infty} \frac{(\log \log n)^{d}}{n \log n} P\left(\left|S_{n}\right| \geq \sqrt{2 V_{n}^{2} \log \log n}\left(\varepsilon+\alpha_{n}\right)\right)
$$




$$
=\frac{1}{\sqrt{\pi}(1+d)} \Gamma(d+3 / 2),
$$

where $\Gamma(\cdot)$ is the gamma function.

Theorem C. Let $\left\{X_{i}, i \geq 1\right\}$ be a sequence of i.i.d. nondegenerate, symmetric random variables which is in the domain of attraction of the normal law with mean zero. Suppose $l(x) \leq c_{1} \exp \left(c_{2}(\log x)^{\beta}\right)$ for some $c_{1}>0, c_{2}>0$ and $0 \leq \beta<1$. Moreover, assume that for $a>-1$ and $b>-1 / 2, \alpha_{n}(\varepsilon)$ is $a$ nonnegative function of $\varepsilon$ such that

$$
\alpha_{n}(\varepsilon) \log \log n \rightarrow \tau \text { as } n \rightarrow \infty \text { and } \varepsilon \searrow \sqrt{1+a} .
$$

Then

(1.6)

$$
\begin{aligned}
& \lim _{\varepsilon \searrow \sqrt{1+a}}\left(\varepsilon^{2}-a-1\right)^{b+1 / 2} \sum_{n=1}^{\infty} \frac{(\log n)^{a}(\log \log n)^{b}}{n} P\left(\left|S_{n}\right| \geq \sqrt{2 V_{n}^{2} \log \log n}\left(\varepsilon+\alpha_{n}(\varepsilon)\right)\right) \\
= & \sqrt{\frac{1}{\pi(1+a)}} \exp (-2 \tau \sqrt{1+a}) \Gamma(b+1 / 2),
\end{aligned}
$$

where, $\tau$ is a finite constant.

The purpose of the present paper is devoted to study the almost sure convergence versions of Theorem $\mathrm{B}$ and Theorem $\mathrm{C}$. In what follows, we denote $I\{\cdot\}$ the indicator function and $C$ the positive constant whose value can differ from line to line, and $C_{1}$ and $C_{2}$ stand for two universal constants respectively. Now, we give our main results.

Theorem 1.1. Suppose the assumptions of Theorem B are satisfied, and in addition, if there exists a $\delta_{0}>1 / 2$ such that

$$
n P\left(\left|X_{1}\right|>\eta_{n}\right) \leq C_{1}(\log \log n)^{-\left(2 d+2+\delta_{0}\right)}
$$

for some $d>-1$, where $\eta_{n}$ is defined in (2.1) and (2.2) below. Then we have

$$
\begin{aligned}
& \lim _{\varepsilon \searrow 0} \varepsilon^{2(d+1)} \sum_{n=1}^{\infty} \frac{(\log \log n)^{d}}{n \log n} I\left\{M_{n} / V_{n} \geq \sqrt{2 \log \log n}\left(\varepsilon+\alpha_{n}\right)\right\} \\
= & \frac{2}{\sqrt{\pi}(1+d)} \Gamma(d+3 / 2) \sum_{k=0}^{\infty} \frac{(-1)^{k}}{(2 k+1)^{2 d+2}} \text { a.s. }
\end{aligned}
$$

and

$$
\begin{aligned}
& \lim _{\varepsilon \searrow 0} \varepsilon^{2(d+1)} \sum_{n=1}^{\infty} \frac{(\log \log n)^{d}}{n \log n} I\left\{\left|S_{n}\right| / V_{n} \geq \sqrt{2 \log \log n}\left(\varepsilon+\alpha_{n}\right)\right\} \\
= & \frac{1}{\sqrt{\pi}(1+d)} \Gamma(d+3 / 2) \text { a.s. }
\end{aligned}
$$

Theorem 1.2 Under the assumptions of Theorem $C$ with $a>-1, b>-1 / 2$ replaced by $-1<a<-1 / 2, b>-1 / 2$ or $a=-1 / 2,-1 / 2<b<-1 / 4$, and in 
addition, if there exists a $\delta_{0}^{\prime}>0$ such that

$$
n P\left(\left|X_{1}\right|>\eta_{n}\right) \leq C_{2}(\log n)^{-(2 a+2)}(\log \log n)^{-\left(2 b+1+\delta_{0}^{\prime}\right)}
$$

for above $a$ and $b$. Then we have

$$
\begin{aligned}
& \lim _{\varepsilon \searrow \sqrt{1+a}}\left(\varepsilon^{2}-a-1\right)^{b+1 / 2} \sum_{n=1}^{\infty} \frac{(\log n)^{a}(\log \log n)^{b}}{n} I\left\{\left(\left|S_{n}\right| / V_{n} \geq \sqrt{2 \log \log n}\left(\varepsilon+\alpha_{n}(\varepsilon)\right)\right\}\right. \\
= & \sqrt{\frac{1}{\pi(1+a)}} \exp (-2 \tau \sqrt{1+a}) \Gamma(b+1 / 2) \text { a.s. },
\end{aligned}
$$

where, $\tau$ is the same finite constant as that in Theorem $C$.

\section{Proofs}

One of the ideas of proofs is based on the truncated random variables. That is, let

$$
l(x)=\mathrm{E}\left(X_{1}^{2} I\left\{\left|X_{1}\right| \leq x\right\}\right), b=\inf \{x \geq 1: l(x)>0\},
$$

and

$$
\eta_{j}=\inf \left\{s: s \geq b+1, \frac{l(s)}{s^{2}} \leq \frac{(\log \log j)^{2}}{j}\right\} \text { for } j=1,2,3, \ldots
$$

Then it is easy to see $\eta_{n}^{2}(\log \log n)^{2} \approx n l\left(\eta_{n}\right)$. For each $n$ and $1 \leq i \leq n$, we denote

(2.3) $\bar{X}_{n i}=X_{i} I\left\{\left|X_{i}\right| \leq \eta_{n}\right\}, \bar{S}_{n}=\sum_{i=1}^{n} \bar{X}_{n i}, \bar{M}_{n}=\max _{1 \leq i \leq n}\left|\bar{S}_{i}\right|, \bar{V}_{n}^{2}=\sum_{i=1}^{n} \bar{X}_{n i}^{2}$.

Furthermore, the following lemma is taken from Csörgö, Szyszkowicz and Wang [4] and will be used in the proofs.

Lemma 2.1. Let $X$ be a random variable, and denote $l(x)=E X^{2} I\{|X| \leq x\}$. The following statements are equivalent:

a) $X$ is in the domain of attraction of the normal law,

b) $x^{2} P(|X|>x)=o(l(x))$,

c) $x E(|X| I\{|X|>x\})=o(l(x))$,

d) $E\left(|X|^{n} I\{|X| \leq x\}\right)=o\left(x^{n-2} l(x)\right)$ for $n>2$.

Next, we give the following lemma which is also useful for the proofs of theorems and is stated as follows.

Lemma 2.2. Let $d>-1$ and $\left\{X_{i}, i \geq 1\right\}$ be a sequence of i.i.d. nondegenerate random variables which is in the domain of attraction of the normal law with mean zero. Suppose $f(\cdot)$ is a real function such that $\sup _{x \in R}|f(x)| \leq C$ 
and $\sup _{x \in R}\left|f^{\prime}(x)\right| \leq C$, and in addition, if there exists a $\delta_{0}>0$ such that $n P\left(\left|X_{1}\right|>\eta_{n}\right) \leq C(\log \log n)^{-\left(2 d+2+\delta_{0}\right)}$. Then for $l \geq m \geq 1$, we have

$$
\left\{\begin{array}{l}
\operatorname{Var}\left(\sum_{n=m}^{l} \frac{(\log \log n)^{d}}{n \log n} f\left(\frac{\bar{M}_{n}}{\sqrt{2 n l\left(\eta_{n}\right) \log \log n}\left(\varepsilon+\alpha_{n}\right)}\right)\right) \leq C \frac{(\log \log m)^{2 d+1 / 2}}{\left(\varepsilon+\alpha_{m}\right) \log m} \\
\operatorname{Var}\left(\sum_{n=m}^{l} \frac{(\log \log n)^{d}}{n \log n} f\left(\frac{\bar{S}_{n}}{\sqrt{2 n l\left(\eta_{n}\right) \log \log n}\left(\varepsilon+\alpha_{n}\right)}\right)\right) \leq C \frac{(\log \log m)^{2 d+1 / 2}}{\left(\varepsilon+\alpha_{m}\right) \log m} \\
\operatorname{Var}\left(\sum_{n=m}^{l} \frac{(\log \log n)^{d}}{n \log n} f\left(\frac{\bar{V}_{n}^{2}}{n l\left(\eta_{n}\right)}\right)\right) \leq C \frac{(\log \log m)^{2 d}}{\log m} \\
\operatorname{Var}\left(\sum_{n=m}^{l} \frac{(\log \log n)^{d}}{n \log n} \sum_{i=1}^{n} I\left\{\left|X_{i}\right| \geq \eta_{n}\right\}\right) \leq \frac{C}{(\log \log m)^{\delta_{0}}}
\end{array}\right.
$$

Proof. Denote $\bar{M}_{i j}=\max _{i \leq k<j}\left|\bar{S}_{k}-\bar{S}_{i}\right|$. Obviously, $\bar{M}_{i j}$ is independent of $\bar{M}_{i}$. Using $\mathrm{E} X=0, \eta_{n}^{2}(\log \log n)^{2} \approx n l\left(\eta_{n}\right)$, Hölder inequality and Lemma 2.1, for $i<j$ we have

$$
\begin{aligned}
& \operatorname{Cov}\left(f\left(\frac{\bar{M}_{i}}{\sqrt{2 i l\left(\eta_{i}\right) \log \log i}\left(\varepsilon+\alpha_{i}\right)}\right), f\left(\frac{\bar{M}_{j}}{\sqrt{2 j l\left(\eta_{j}\right) \log \log j}\left(\varepsilon+\alpha_{j}\right)}\right)\right) \\
= & \operatorname{Cov}\left(f\left(\frac{\bar{M}_{i}}{\sqrt{2 i l\left(\eta_{i}\right) \log \log i}\left(\varepsilon+\alpha_{i}\right)}\right)\right. \\
& \left.f\left(\frac{\bar{M}_{j}}{\sqrt{2 j l\left(\eta_{j}\right) \log \log j}\left(\varepsilon+\alpha_{j}\right)}\right)-f\left(\frac{\bar{M}_{i j}}{\sqrt{2 j l\left(\eta_{j}\right) \log \log j}\left(\varepsilon+\alpha_{j}\right)}\right)\right) \\
\leq & C \mathrm{E} \mid f\left(\frac{\bar{M}_{j}}{\sqrt{2 j l\left(\eta_{j}\right) \log \log j}\left(\varepsilon+\alpha_{j}\right)}-f\left(\frac{\bar{M}_{i j}}{\sqrt{2 j l\left(\eta_{j}\right) \log \log j}\left(\varepsilon+\alpha_{j}\right)}\right) \mid\right. \\
\leq & C \frac{\mathrm{E} \bar{M}_{i}}{\sqrt{2 j l\left(\eta_{j}\right) \log \log j}\left(\varepsilon+\alpha_{j}\right)} \\
\leq & C \frac{\mathrm{E} \max x_{1 \leq k \leq i}\left|\bar{S}_{k}-\mathrm{E} \bar{S}_{k}\right|+i \mathrm{E}\left|X_{1}\right| I\left\{\left|X_{1}\right|>\eta_{i}\right\}}{\sqrt{2 j l\left(\eta_{j}\right) \log \log j\left(\varepsilon+\alpha_{j}\right)}} \\
\leq & C \frac{\sqrt{i l\left(\eta_{i}\right)}+o\left(i l\left(\eta_{i}\right) / \eta_{i}\right)}{\sqrt{2 j l\left(\eta_{j}\right) \log \log j}\left(\varepsilon+\alpha_{j}\right)} \\
\leq & C \frac{\sqrt{i}}{\sqrt{j \log \log j\left(\varepsilon+\alpha_{j}\right)}+o\left(\frac{\sqrt{i} \log \log i}{\sqrt{j \log \log j}\left(\varepsilon+\alpha_{j}\right)}\right)}
\end{aligned}
$$

which yields

$$
\begin{aligned}
& \operatorname{Var}\left(\sum_{n=m}^{l} \frac{(\log \log n)^{d}}{n \log n} f\left(\frac{\bar{M}_{n}}{\sqrt{2 n l\left(\eta_{n}\right) \log \log n}\left(\varepsilon+\alpha_{n}\right)}\right)\right) \\
\leq & C \sum_{n=m}^{l} \frac{(\log \log n)^{2 d}}{n^{2}(\log n)^{2}}+2 \sum_{i=m}^{l-1} \sum_{j=i+1}^{l} \frac{(\log \log i)^{d}}{i \log i} \frac{(\log \log j)^{d}}{j \log j} \\
& \left(\frac{C \sqrt{i}}{\sqrt{j \log \log j}\left(\varepsilon+\alpha_{j}\right)}+o\left(\frac{\sqrt{i} \log \log i}{\sqrt{j \log \log j}\left(\varepsilon+\alpha_{j}\right)}\right)\right)
\end{aligned}
$$




$$
\begin{aligned}
& \leq C \frac{(\log \log m)^{2 d}}{m(\log m)^{2}}+C \frac{(\log \log m)^{2 d-1 / 2}}{\left(\varepsilon+\alpha_{m}\right) \log m}+o\left(\frac{(\log \log m)^{2 d+1 / 2}}{\left(\varepsilon+\alpha_{m}\right) \log m}\right) \\
& \leq C \frac{(\log \log m)^{2 d+1 / 2}}{\left(\varepsilon+\alpha_{m}\right) \log m} .
\end{aligned}
$$

The first part of (2.4) is proved. Similarly, the second and the third parts of (2.4) can be proved by the same arguments, and we omit the details here. Consider the fourth part of (2.4) now. In view of the assumption $n \mathrm{P}\left(\left|X_{1}\right|>\right.$ $\left.\eta_{n}\right) \leq C_{1}(\log \log n)^{-\left(2 d+2+\delta_{0}\right)}$ for some $\delta_{0}>0$, we have

$$
\begin{aligned}
& \operatorname{Var}\left(\sum_{n=m}^{l} \frac{(\log \log n)^{d}}{n \log n} \sum_{i=1}^{n} I\left\{\left|X_{i}\right| \geq \eta_{n}\right\}\right) \\
\leq & C \sum_{n=m}^{l} \frac{(\log \log n)^{2 d}}{n^{2}(\log n)^{2}} \cdot n \mathrm{P}\left(\left|X_{1}\right| \geq \eta_{n}\right) \\
& +2 \sum_{j=m+1}^{l} \sum_{i=m}^{j-1} \frac{(\log \log i)^{d}}{i \log i} \frac{(\log \log j)^{d}}{j \log j} \operatorname{Cov}\left(\sum_{k=1}^{i} I\left\{\left|X_{k}\right| \geq \eta_{i}\right\}, \sum_{k=1}^{j} I\left\{\left|X_{k}\right| \geq \eta_{j}\right\}\right) \\
\leq & C \sum_{n=m}^{l} \frac{(\log \log n)^{2 d}}{n^{2}(\log n)^{2}} \cdot(\log \log n)^{-\left(2 d+2+\delta_{0}\right)} \\
& +2 \sum_{j=m+1}^{l} \sum_{i=m}^{j-1} \frac{(\log \log i)^{d}}{i \log i} \frac{(\log \log j)^{d}}{j \log j} \cdot i \mathrm{P}\left(\left|X_{1}\right| \geq \eta_{i}\right) \\
\leq & C \frac{(\log \log m)^{-2-\delta_{0}}}{m(\log m)^{2}}+C \sum_{j=m+1}^{l} \sum_{i=m}^{j-1} \frac{(\log \log i)^{d}}{i \log i} \frac{(\log \log j)^{d}}{j \log j} \cdot(\log \log i)^{-\left(2 d+2+\delta_{0}\right)} \\
\leq & \frac{C}{(\log \log m)^{\delta_{0}}} .
\end{aligned}
$$

Proofs of Theorem 1.1 and Theorem 1.2. We only show (1.7), since (1.8) and (1.9) can be proved by the same way. We first give an upper and a lower bound for the indicator function of self-normalized partial sums. For any given $0<\varepsilon<1$, it is clear that

$$
\begin{aligned}
I\left\{\frac{M_{k}}{V_{k}} \geq x\right\} \leq & I\left\{\frac{\bar{M}_{k}}{\sqrt{\left.(1-\varepsilon) k l\left(\eta_{k}\right)\right)}} \geq x\right\} \\
& +I\left\{\bigcup_{i=1}^{k}\left\{\left|X_{i}\right|>\eta_{k}\right\}\right\}+I\left\{\bar{V}_{k}^{2}<(1-\varepsilon) k l\left(\eta_{k}\right)\right\}
\end{aligned}
$$

and

$$
I\left\{\frac{M_{k}}{V_{k}} \geq x\right\} \geq I\left\{\frac{\bar{M}_{k}}{\sqrt{\left.(1+\varepsilon) k l\left(\eta_{k}\right)\right)}} \geq x\right\}
$$




$$
-I\left\{\bigcup_{i=1}^{k}\left\{\left|X_{i}\right|>\eta_{k}\right\}\right\}-I\left\{\bar{V}_{k}^{2}>(1+\varepsilon) k l\left(\eta_{k}\right)\right\} .
$$

Denote $B(\varepsilon)=\exp \left(\exp \left(1 /\left(\varepsilon^{2} M\right)\right)\right)$ for any $M>0$. Fix $M>4$ and $0<$ $\delta_{1}<1 / 2$, and let $f(\cdot)$ be a real function, such that $I\left\{|x| \geq \sqrt{1-\delta_{1}}\right\} \leq f(x) \leq$ $I\left\{|x| \geq 1-\delta_{1}\right\}, \sup _{x}\left|f^{\prime}(x)\right|<\infty$. Define $\varepsilon_{k}=1 / k, k \geq 4 M+1$. Then, it follows from Lemma 2.2 and noting that $\alpha_{n}=O(1 / \log \log n)=O\left(M / k^{2}\right)=o\left(\varepsilon_{k}\right)$ for $n>B\left(\varepsilon_{k}\right)$,

$$
\begin{aligned}
& \operatorname{Var}\left(\sum_{n>B\left(\varepsilon_{k}\right)} \frac{(\log \log n)^{d}}{n \log n} f\left(\frac{\bar{M}_{n}}{\sqrt{2 n l\left(\eta_{n}\right) \log \log n}\left(\varepsilon_{k}+\alpha_{n}\right)}\right)\right) \\
\leq & C \frac{\left(\log \log B\left(\varepsilon_{k}\right)\right)^{2 d+1 / 2}}{\varepsilon_{k} \log B\left(\varepsilon_{k}\right)} \\
\leq & C M^{-(2 d+1 / 2)} k^{4 d+2} e^{-4 k},
\end{aligned}
$$

which together with Borel-Cantelli lemma yields

$$
\begin{aligned}
& \sum_{n>B\left(\varepsilon_{k}\right)} \frac{(\log \log n)^{d}}{n \log n}\left(f\left(\frac{\bar{M}_{n}}{\sqrt{2 n l\left(\eta_{n}\right) \log \log n}\left(\varepsilon_{k}+\alpha_{n}\right)}\right)\right. \\
& \left.-\operatorname{E} f\left(\frac{\bar{M}_{n}}{\sqrt{2 n l\left(\eta_{n}\right) \log \log n}\left(\varepsilon_{k}+\alpha_{n}\right)}\right)\right) \longrightarrow 0 \text { a.s. }
\end{aligned}
$$

Similarly, one has

$$
\sum_{n>B\left(\varepsilon_{k}\right)} \frac{(\log \log n)^{d}}{n \log n}\left(f\left(\frac{\bar{V}_{n}^{2}}{n l\left(\eta_{n}\right)}\right)-\mathrm{E} f\left(\frac{\bar{V}_{n}^{2}}{n l\left(\eta_{n}\right)}\right)\right) \longrightarrow 0 \text { a.s. }
$$

By means of the similar arguments and using the assumption $\delta_{0}>1 / 2$, one also has

$$
\sum_{n>B\left(\varepsilon_{k}\right)} \frac{(\log \log n)^{d}}{n \log n}\left(\sum_{i=1}^{n} I\left\{\left|X_{i}\right| \geq \eta_{n}\right\}-n \mathrm{P}\left(\left|X_{1}\right| \geq \eta_{n}\right)\right) \longrightarrow 0 \text { a.s. }
$$

since

$$
\operatorname{Var}\left(\sum_{n>B\left(\varepsilon_{k}\right)} \frac{(\log \log n)^{d}}{n \log n} \sum_{i=1}^{n} I\left\{\left|X_{i}\right| \geq \eta_{n}\right\}\right) \leq \frac{C M^{\delta_{0}}}{k^{2 \delta_{0}}} .
$$

Using the inequality (2.8), we have

$$
\begin{aligned}
& \lim _{\varepsilon \searrow 0} \varepsilon^{2(d+1)} \sum_{n>B(\varepsilon)} \frac{(\log \log n)^{d}}{n \log n} I\left\{M_{n} / V_{n} \geq \sqrt{2 \log \log n}\left(\varepsilon+\alpha_{n}\right)\right\} \\
\leq & \limsup _{k \rightarrow \infty} \varepsilon_{k-1}^{2(d+1)} \sum_{n>B\left(\varepsilon_{k}\right)} \frac{(\log \log n)^{d}}{n \log n} I\left\{M_{n} / V_{n} \geq \sqrt{2 \log \log n}\left(\varepsilon_{k}+\alpha_{n}\right)\right\}
\end{aligned}
$$




$$
\begin{aligned}
\leq & \limsup _{k \rightarrow \infty} \varepsilon_{k-1}^{2(d+1)} \sum_{n>B\left(\varepsilon_{k}\right)} \frac{(\log \log n)^{d}}{n \log n}\left(I\left\{\bar{M}_{n} / \sqrt{\left(1-\delta_{1}\right) n l\left(\eta_{n}\right)} \geq \sqrt{2 \log \log n}\left(\varepsilon_{k}+\alpha_{n}\right)\right\}\right. \\
& \left.+I\left\{\bigcup_{i=1}^{n}\left\{\left|X_{i}\right| \geq \eta_{n}\right\}\right\}+I\left\{\bar{V}_{n}^{2} /\left(n l\left(\eta_{n}\right)\right)<\left(1-\delta_{1}\right)\right\}\right) \\
=: & \limsup _{k \rightarrow \infty} \varepsilon_{k-1}^{2(d+1)} \sum_{n>B\left(\varepsilon_{k}\right)} \frac{(\log \log n)^{d}}{n \log n}\left(I_{1}+I_{2}+I_{3}\right) .
\end{aligned}
$$

We are going on to treat with the three terms above respectively. Together (2.11) with Propositions 3.1 and 3.2 in Pang, Zhang and Wang [11] yields

$$
\begin{aligned}
& \limsup _{k \rightarrow \infty} \varepsilon_{k-1}^{2(d+1)} \sum_{n>B\left(\varepsilon_{k}\right)} \frac{(\log \log n)^{d}}{n \log n} I_{1} \\
& \leq \limsup _{k \rightarrow \infty} \varepsilon_{k-1}^{2(d+1)} \sum_{n>B\left(\varepsilon_{k}\right)} \frac{(\log \log n)^{d}}{n \log n} f\left(\frac{\bar{M}_{n}}{\sqrt{2 n l\left(\eta_{n}\right) \log \log n}\left(\varepsilon_{k}+\alpha_{n}\right)}\right) \\
& \leq\left.\limsup _{k \rightarrow \infty} \varepsilon_{k-1}^{2(d+1)} \sum_{n>B\left(\varepsilon_{k}\right)} \frac{(\log \log n)^{d}}{n \log n} \mathrm{E} f\left(\frac{\bar{M}_{n}}{\sqrt{2 n l\left(\eta_{n}\right) \log \log n}\left(\varepsilon_{k}+\alpha_{n}\right)}\right)\right) \\
& \leq \limsup _{k \rightarrow \infty} \varepsilon_{k-1}^{2(d+1)} \sum_{n>B\left(\varepsilon_{k}\right)} \frac{(\log \log n)^{d}}{n \log n} \\
& \cdot \mathrm{P}\left(\frac{M}{n}_{\sqrt{n l\left(\eta_{n}\right)}} \geq\right. \\
&\left.\left(1-\delta_{1}\right) \sqrt{2 \log \log n}\left(\varepsilon_{k}+\alpha_{n}\right)\right) \\
& \leq\left(1-\delta_{1}\right)^{-2(d+1)} \frac{2}{\sqrt{\pi}(1+d)} \Gamma(d+3 / 2) \sum_{k=0}^{\infty} \frac{(-1)^{k}}{(2 k+1)^{2 d+2}} \text { a.s. }
\end{aligned}
$$

For the second term of (2.15), in view of (2.13) we have

$$
\begin{aligned}
& \limsup _{k \rightarrow \infty} \varepsilon_{k-1}^{2(d+1)} \sum_{n>B\left(\varepsilon_{k}\right)} \frac{(\log \log n)^{d}}{n \log n} I_{2} \\
\leq & \limsup _{k \rightarrow \infty} \varepsilon_{k-1}^{2(d+1)} \sum_{n>B\left(\varepsilon_{k}\right)} \frac{(\log \log n)^{d}}{n \log n} \sum_{i=1}^{n} I\left\{\left|X_{i}\right| \geq \eta_{n}\right\} \\
\leq & \limsup _{k \rightarrow \infty} \varepsilon_{k-1}^{2(d+1)} \sum_{n=1}^{\infty} \frac{(\log \log n)^{d}}{\log n} \mathrm{P}\left(\left|X_{1}\right| \geq \sqrt{1-\delta_{1}} \eta_{n}\right)=0 \text { a.s. }
\end{aligned}
$$

where in the equation above, we have used the similar arguments for showing the right hand of (3.10) in Pang, Zhang and Wang [11]. Similarly, by using the same arguments appeared above, (2.12) and the inequality (3.13) in Pang, 
Zhang and Wang [11], we have

$$
\limsup _{k \rightarrow \infty} \varepsilon_{k-1}^{2(d+1)} \sum_{n>B\left(\varepsilon_{k}\right)} \frac{(\log \log n)^{d}}{n \log n} I_{3}=0 \text { a.s. }
$$

On the other hand,

$$
\begin{aligned}
& \limsup _{\varepsilon \searrow 0} \varepsilon^{2(d+1)} \sum_{n \leq B(\varepsilon)} \frac{(\log \log n)^{d}}{n \log n} I\left\{M_{n} / V_{n} \geq \sqrt{2 \log \log n}\left(\varepsilon+\alpha_{n}\right)\right\} \\
\leq & \limsup _{\varepsilon \searrow 0} \varepsilon^{2(d+1)} \sum_{n \leq B(\varepsilon)} \frac{(\log \log n)^{d}}{n \log n} \\
\leq & C \limsup _{\varepsilon \searrow 0} \varepsilon^{2(d+1)} \int_{e^{e}}^{B(\varepsilon)} \frac{(\log \log x)^{d}}{x \log x} d x \\
\leq & \frac{C}{1+d} \limsup _{\varepsilon \searrow 0} \varepsilon^{2(d+1)}(\log \log B(\varepsilon))^{d+1} \\
(2.19)= & \frac{C}{1+d} \cdot \frac{1}{M^{d+1}} .
\end{aligned}
$$

Now, it follows from (2.15)-(2.19) and by the arbitrarinesses of $\delta_{1}$ and $M$, we have

$$
\begin{aligned}
& \limsup _{\varepsilon \searrow 0} \varepsilon^{2(d+1)} \sum_{n=1}^{\infty} \frac{(\log \log n)^{d}}{n \log n} I\left\{M_{n} / V_{n} \geq \sqrt{2 \log \log n}\left(\varepsilon+\alpha_{n}\right)\right\} \\
& \leq \frac{2}{\sqrt{\pi}(1+d)} \Gamma(d+3 / 2) \sum_{k=0}^{\infty} \frac{(-1)^{k}}{(2 k+1)^{2 d+2}} \text { a.s. }
\end{aligned}
$$

The similar arguments also yield

$$
\begin{aligned}
& \liminf _{\varepsilon \searrow 0} \varepsilon^{2(d+1)} \sum_{n=1}^{\infty} \frac{(\log \log n)^{d}}{n \log n} I\left\{M_{n} / V_{n} \geq \sqrt{2 \log \log n}\left(\varepsilon+\alpha_{n}\right)\right\} \\
& \geq \frac{2}{\sqrt{\pi}(1+d)} \Gamma(d+3 / 2) \sum_{k=0}^{\infty} \frac{(-1)^{k}}{(2 k+1)^{2 d+2}} \text { a.s., }
\end{aligned}
$$

we omit the details here. The proofs are now completed.

\section{References}

[1] L. E. Baum and M. Katz, Convergence rates in the law of large numbers, Trans. Amer. Math. Soc. 120 (1965), 108-123.

[2] R. Chen, A remark on the tail probability of a distribution, J. Multivariate Anal. 8 (1978), no. 2, 328-333.

[3] M. Csörgö and P. Révész, Strong Approximations in Probability and Statistics, Academic, New York, 1981.

[4] M. Csörgö, B. Szyszkowicz, and Q. Y. Wang, Donsker's theorem for self-normalized partial sums processes, Ann. Probab. 31 (2003), no. 3, 1228-1240. 
[5] V. H. de la Peña, T. L. Lai, and Q. M. Sha, Self-Normalized Processes: Limit Theory and Statistical Applications, Springer, New York, 2009.

[6] P. Erdős, On a theorem of Hsu and Robbins, Ann. Math. Statist. 20 (1949), 286-291.

[7] A. Gut and A. Spătaru, Precise asymptotics in the law of the iterated logarithm, Ann. Probab. 28 (2000), no. 4, 1870-1883.

[8] C. C. Heyde, A supplement to the strong law of large numbers, J. Appl. Probab. 12 (1975), 173-175.

[9] P. L. Hsu and H. Robbins, Complete convergence and the law of large numbers, Proc. Natl. Acad. Sci. U.S.A. 33 (1947), 25-31.

[10] T. L. Lai and Q. M. Shao, Self-normalized limit theorems in probability and statistics. In: Asymptotic Theory in Probability and Statistics with Applications (Editors: T. L. Lai, L. F. Qian, and Q. M. Shao), International Press of Boston. pp. 3-43, 2007.

[11] T. X. Pang, L. X. Zhang, and J. F. Wang, Precise asymptotics in the self-normalized law of the iterated logarithm, J. Math. Anal. Appl. 340 (2008), no. 2, 1249-1262.

[12] Q. M. Shao, Self-normalized large deviations, Ann. Probab. 25 (1997), no. 1, 285-328.

[13] A. Spătaru, Precise asymptotics in Spitzer's law of large numbers, J. Theoret. Probab. 12 (1999), no. 3, 811-819.

[14] L. X. Zhang, Precise rates in the law of the iterated logarithm, Available at http:// arxiv1.library. cornell.edu/abs/math/0610519v1.

Department of Mathematics

YUQUAN CAMPUS

ZHEJIANG UNIVERSITY

Hangzhou 310027, P. R. China

E-mail address: txpang@zju.edu.cn 